This is an author produced version of a paper published in International Journal of Biochemistry and Cell Biology. This paper has been peer-reviewed but does not include the final publisher proof-corrections or journal pagination.

Citation for the published paper:

Ekberg, Jenny and Brunhoff, Cecilia and Jaras, Marcus and

Fan, Xiaolong and Landberg, Goran and Persson, Jenny Liao

"Increased expression of cyclin A1 protein is associated with all-trans retinoic acid-induced apoptosis"

International Journal of Biochemistry and Cell Biology, 2006, Issue: Feb 21

http://dx.doi.org/10.1016/j.biocel.2006.01.011

Access to the published version may require journal subscription.

Published with permission from: Elsevier 


\section{Increased expression of cyclin A1 protein is associated with all-trans retinoic acid-induced apoptosis}

Jenny Ekberg ${ }^{1}$, Cecilia Brunhoff ${ }^{1}$, Marcus Järås², Xiaolong Fan², Göran Landberg ${ }^{1}$, Jenny Liao Persson ${ }^{1 *}$

${ }^{1}$ Division of Pathology, Department of Laboratory Medicine, Lund University, University Hospital, 20502 Malmö, Sweden. ${ }^{2}$ Division of Molecular Medicine and Gene Therapy, Department of Laboratory Medicine, Lund University, 22184 Lund, Sweden.

* Reprint requests should be addressed at the Division of Pathology, Department of Laboratory Medicine, Lund University, University Hospital, 205 02, Malmö, Sweden. Tel: 46-40-338391, Fax: 46-40-337063. E-mail: Jenny_L.Persson@med.lu.se

\section{Abstract}

Deregulated cell growth and inhibition of apoptosis are hallmarks of cancer. All-trans retinoic acid induces clinical remission in patients with acute promyelocytic leukemia by inhibiting cell growth and inducing differentiation and apoptosis of the leukemic blasts. An important role of the cell cycle regulatory protein cyclin A1 in the development of acute myeloid leukemia has previously been demonstrated in a transgenic mouse model. We have recently shown that there was a direct interaction between cyclin A1 and a major alltrans retinoic acid receptor, RAR $\alpha$, following all-trans retinoic acid treatment of leukemic cells. In the present study, we investigated whether cyclin A1 might be involved in all-trans retinoic acid induced apoptosis in U-937 leukemic cells. We found that all-trans retinoic acid induced apoptosis was associated with concomitant increase in cyclin A1 expression. However, there was no induction of cyclin A1 mRNA expression following the all-trans retinoic acid induced apoptosis. Treatment of cells with a caspase inhibitor was not able to prevent all-trans retinoic acid-induced up-regulation of cyclin A1 expression. Interestingly, induced cyclin A1 expression in U-937 cells led to a significant increase in the proportion of apoptotic cells. Further, U-937 cells overexpressing cyclin A1 appeared to be more sensitive to all-trans retinoic acid-induced apoptosis indicating the ability of cyclin A1 to mediate all-trans retinoic acid induced apoptosis. Induced cyclin E expression was not able to initiate cell death in U-937 cells. Our results indicate that cyclin A1 might have a role in apoptosis by mediating all-trans retinoic acid induced apoptosis. 


\section{Introduction}

All-trans Retinoic Acid (ATRA) has proven to be an effective agent in the clinical treatment of patients with acute promyelocytic leukemia (He et al., 1999). ATRA treatment results in an inhibition of growth of leukemic cells, which is associated with G0/G1 arrest and changes in expression of cell cycle regulatory proteins (Dimberg et al., 2002). In addition, ATRA triggers apoptosis in leukemic cells (Monczak et al., 1997). Apoptosis is an important mechanism for regulating the amount of hematopoietic stem cells and maintenance of cellular homeostasis (Passegue et al., 2003). Defects in apoptosis may allow oncogenic activation and genetic instability to persist (Puccetti \& Ruthardt, 2004). The mechanism by which ATRA induces apoptosis in leukemic cells is poorly understood although it has been suggested that ATRA induces apoptosis through activation of its ligand RXR (Mehta et al., 1996). A decrease in Bcl-XL expression and an activation of caspase- 3 and caspase- 6 have been observed in ATRA-induced apoptotic cells. Further, ATRA treatment results in a decrease in mitochondrial membrane potential which is accompanied with DNA fragmentation (Fujimura et al., 2003) suggesting that ATRA induces apoptosis through mitochondrialmediated pathways.
Cell cycle control plays a fundamental role in cell differentiation and growth. The proper regulation of the cell cycle machinery, including cyclins, cyclin dependent kinases (CDK), and the negative regulators $\mathrm{CDK}$ inhibitors (CDKI), is critical for hematopoiesis (Dao \& Nolta, 1999; Furukawa, 1998). The cyclins are usually classified into two categories, those that function at G1/S, including the D-type cyclins and cyclin E, and the mitotic cyclins, primarily the B-type cyclins, that induce the G2/M transition (Roberts, 1999). The A-type cyclin, cyclin A2, is believed to function at both G1/S and G2/M phase transitions (Cardoso et al., 1993). In addition to their role in cell proliferation, cyclins and CDKs has also been found to be associated with apoptosis. For instance, declined expression of cyclin D has been shown in apoptotic cells induced by TNF $\alpha$ and was a result of ubiquitin/ proteasome-dependent degradation (Hu et al., 2002). Inhibition of CDK1 activity has led to an increasing level of subsequent apoptosis induced by DNA-damaging drugs (Ongkeko et al., 1995), suggesting that CDK1 has a function in the suppression of cell death. It has also been shown that CDK2 activity is associated with panaxadiol induced apoptotic cell death and correlates with depolarization of mitochondrial membrane potential (Jin et al., 2003). 
The cell cycle regulatory protein cyclin A1 is expressed in early hematopoietic cells (Yang et al., 1999) and male germ cells. Cyclin A1 has been demonstrated to be required for $\mathrm{G} 2 / \mathrm{M}$ phase transition in meiotic division of male germ cells by gene targeting (Liu et al., 2000; Liu et al., 1998). Expression of cyclin A1 is altered in patients with acute myeloid leukemia (Yang et al., 1999) and patients with male germ cell tumors (Liao et al., 2004). Targeted overexpression of cyclin A1 in early myeloid cells has been shown to initiate acute myeloid leukemia (AML) in a transgenic mouse model (Liao et al., 2001). We have recently shown that cyclin A1/RAR $\alpha / C D K 1$ complexes formed following treatment with ATRA, suggesting that the interaction between cyclin A1 and RAR $\alpha$ is ATRAdependent (Ekberg et al., 2004). The aim of this study was therefore to investigate the expression of cyclin A1 in response to ATRA treatment in detail and to analyze whether cyclin A1 itself could mediate apoptosis. We now show that induction of apoptosis by ATRA led to an increase in the expression of cyclin A1 protein but not cyclin A1 mRNA expression. Cyclin A1 overexpression promoted apoptosis in U-937 and T47D cells and cyclin A1 was able to enhance the effect of ATRA on apoptosis and cell growth inhibition. This was in contrast to what was observed in cells overexpressing cyclin E, which did not result in increased cell death. Our results indicate that cyclin A1 expression is correlated with cell cycle arrest and apoptosis induced by ATRA.

\section{Materials and methods}

Cell culture, transfection and drug treatment

Both the human leukemic cell line U937 (monoblast) and the human breast cancer cell line T47D was maintained in RPMI 1640 medium (PAA Laboratories, Pashing, Austria) supplemented with $10 \%$ fetal calf serum (FCS) (Sigma, St. Louis, MO). In addition, T47D cells were supplemented with $0.6 \mathrm{ml} / 1$ insulin. For transfection, full length cyclin A1 cDNA was cloned into PCMS-EGFP vector (Clontech, CA). EGFP-C2 vector containing cyclin $\mathrm{E}$ cDNA has previously been described (Loden et al., 2002). Transient tansfection was performed using the electroporation system with Nucleofection kit (AmaxaBiosystems, Gaithersburg, Cologne, Germany) according to the manufacturer's instruction. 5 to 10 g cyclin A1 and cyclin E expression vectors, as well as control EGFP vectors were used. For ATRA treatment, U-937 cells were cultured in the medium containing ATRA (Sigma) dissolved in ethanol. To determine the dose-dependent effect of ATRA on the induction of apoptosis, U-937 cells were cultured in the absence or presence of ATRA at a concentration of $1 \mu \mathrm{M}, 5 \mu \mathrm{M}, 50 \mu \mathrm{M}$ or 
$100 \mu \mathrm{M}$ for $100 \mathrm{~h}$. Control cells were treated with ethanol for the same time period. To determine the timedependent effect of ATRA, U-937 cells were cultured in the absence and presence of ATRA at $100 \mu \mathrm{M}$ for $24 \mathrm{~h}$, $48 \mathrm{~h}$ and $72 \mathrm{~h}$. For treatment with caspase inhibitor, cells were pretreated with $50 \mu \mathrm{M} \mathrm{Z-VAD-FMK}$ (Sigma) for 1 hour before the addition of ATRA and cultured for another 48 hours. To examine whether cyclin A1 is regulated at post-transcriptional level in ATRA-treated cells, cycloheximide (CHX) was used. U-937 cells were cultured in the absence or presence of $100 \mu \mathrm{M}$ ATRA and $1 \mu \mathrm{g} / \mathrm{ml}$ CHX for 48 hours.

\section{Immunoblotting and source of antibodies}

For immunoblotting cells were lysed in ice-cold RIPA lysis buffer $(150 \mathrm{mM}$ $\mathrm{NaCl}, 50 \mathrm{mM}$ Tris-HCL pH 7.5, 1\% NP$40,10 \mathrm{mM} \mathrm{NaF}$ ) and the protease inhibitor cocktail Complete Mini (Roche, Basel, Switzerland) and centrifuged at $12000 \times \mathrm{g}$ for $20 \mathrm{~min}$ at $4^{\mathrm{o}} \mathrm{C}$. The membranes were probed with primary antibodies including monoclonal antibodies against cyclin A1, bcl-2 (PharMingene, San Diego, CA), cyclin B1, (Upstate Biotech Inc., New York, NY), cyclin A2, CDK1, CDK2 (Transduction Laboratory, San Jose, CA), caspase-3 (Alexis Biochemicals, Lausen/Switzerland) or actin (Santa Cruz Biotechnology, Santa Cruz, CA) followed by horseradish peroxidase (HRP)-conjugated secondary antibodies (Amersham Life Science, Alesbury, U.K.) and visualized using the Enhanced ChemiLuminescence detection system (ECL) and ECL films (Amersham Pharmacia Biotech).

\section{Apoptosis analysis}

For Annexin-V staining, cells were washed in $1 \times$ PBS and dissolved in bindning buffer $(0.01 \mathrm{M}$ Hepes $\mathrm{pH} 7.4$, $0.14 \mathrm{M} \mathrm{NaCl}, 2.5 \mathrm{mM} \mathrm{CaCl}_{2}$ ), stained with Annexin V conjugated with APC (PharMingene). The cells were then subjected to flow cytometry analysis using FACS Calibur (BD Biosciencies, San Jose, CA). To label the breaks in genomic DNA characteristic of apoptosis, cells were fixed and embedded in paraffin sections as described previously (Ekberg et al., 2004). Terminal deoxynucleotidyl transferas (TdT)-mediated dUTP nickend-labeling (TUNEL) analysis was then performed by using ApoAlert ${ }^{\circledR}$ DNA Fragmentation Assay Kit according to the manufacturer's instructions (Clontech, Palo Alto, CA). Fluorescein-labeled cells were analyzed under a fluorescence microscope.

\section{RT-PCR}

RNA was prepared with TRIsol ${ }^{\circledR}$ reagent (Total RNA Isolation Reagent) according to the manufacturer's instructions (Life Technologies, 
Rockville, MD). RNA was reverse transcribed to cDNA following the supplier's protocol (Life Tehcnologies). The primer sequences were derived to span exon-exon junctions of human cyclin A1 and GAPDH. Primers for cyclin A1: 5'- GTCAGAGAGGGGAT GGCAT-3' and: 5'-CCAGTCCAC CAGAATCGTG-3' and for GAPDH 5' AACAGCGACACCCACTCCTC-3' and 5'-GGAGGGGAGATTCAGTG TGGT 3'. The PCR conditions used were: an initial 5 min step at $95^{\circ} \mathrm{C}$ was followed by denaturation at $94^{\circ} \mathrm{C}$ for $1 \mathrm{~min}$, annealing at $58^{\circ} \mathrm{C}$ for $1 \mathrm{~min}$, and extension at $70^{\circ} \mathrm{C}$ for $1 \mathrm{~min}$ for 18 cycles using only cyclin A1 primers, then for another 17 cycles after the addition of GAPDH primers and with a final extension step at $70^{\circ} \mathrm{C}$ for $10 \mathrm{~min}$.

\section{Results}

To investigate the effect of ATRA on apoptosis in U-937 cells and to examine the expression of cyclin A1 in response to ATRA-induced cell death, the cells were cultured in the presence of increasing doses of ATRA for 4 days. The proportion of apoptotic cells was measured by Annexin-V staining. As shown in dose-response curve, induction of apoptosis was much more pronounced when cells were treated with ATRA at $100 \mu \mathrm{M}$ ATRA (Fig 1A). It has been shown that caspase-3, a major apoptosis effector, becomes activated during apoptosis
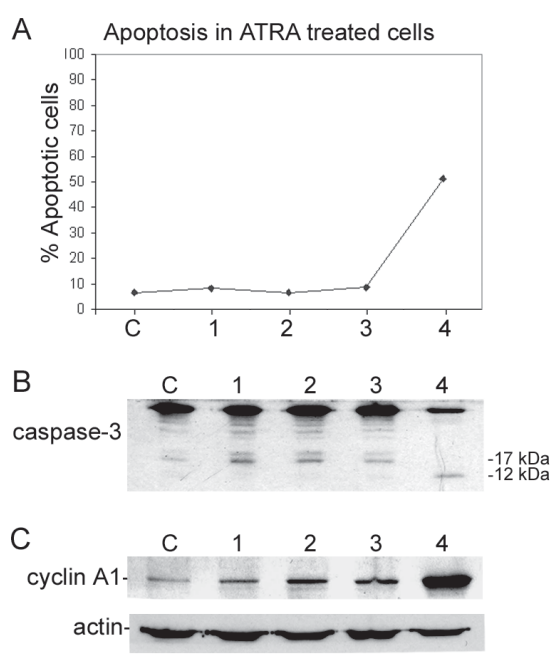

Figure 1. Expression of cyclin A1 and caspase- 3 in response to ATRA-induced apoptosis. (A) Cells were treated with increasing doses of ATRA (1, 5, 50 and $100 \mu \mathrm{M}$ indicated as 1-4) or solvent, the amount of apoptotic cells were measured using Annexin-V staining. " $C$ " indicates sample treated with solvent. (B) Protein extracts were prepared from the cells used in panel A, subjected to immunoblot analysis and probed with antibody against caspase-3. (C) The same blot shown in panel B was probed with antibodies against cyclin A1 and actin.

and its activation correlates with the rate of apoptosis (Tewari et al., 1995). We therefore examined expression of caspase- 3 by immunoblot analysis. We noticed that treatment of U-937 cells with ATRA at higher doses resulted in a marked cleavage of caspase-3 with the appearance of small fragments $(19,17$, and $12 \mathrm{kDa})$ indicating cleavage of the procaspase following cell death in ATRA-treated samples (Fig 1B). Thus, the two 
independent approaches using annexin- $\mathrm{V}$ and caspase-3 for measuring apoptosis provided strong evidence for the induction of apoptosis by ATRA. Expression of cyclin A1 in response to ATRA-induced apoptosis was further determined in U-937 cells by immunoblot analysis (Fig 1C). A significant induction in cyclin A1 expression was detected in ATRAtreated cells as measured by densitometric quantification of cyclin A1 bands on immunoblots from three independent experiments (data not shown). The quantification analysis also confirmed that the concomitant induction of cyclin A1 expression indeed correlated with the rate of apoptosis and the activation of casepase-3.

Cyclin A1 protein level is increased after ATRA induced apoptosis, in a time-dependent manner

To further assess the effect of ATRA induced apoptosis on the expression of cell cycle regulatory proteins, U-937 cells were treated with ATRA for 24, 48 and 72 hours. The increased rate of apoptosis readily started after 48 hours treatment of the cells and a significant proportion of apoptotic cells $(62 \%)$ was observed after 72 hour treatment compared with $20 \%$ apoptotic cells in control sample (Fig 2A). TUNEL-assay, another analysis hallmark of DNA damage was performed to further quantify the amount of apoptotic cells. The
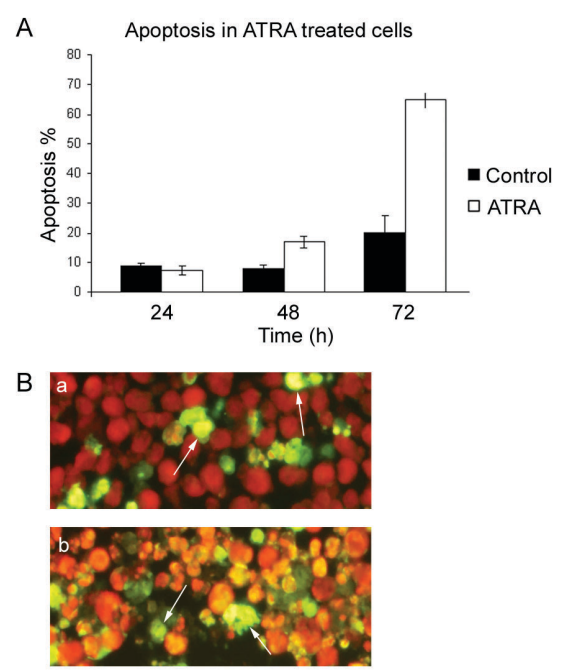

Figure 2. ATRA-induced apoptosis in U937 cells. (A) Cells were treated with 100 $\mu \mathrm{M}$ ATRA or solvent for 24,48 and 72 hours. The proportion of apoptotic cells in ATRA-treated (ATRA) and in solventtreated (C) were measured using Annexin-V staining. (B) TUNEL assay of control cells (a) and cells treated with ATRA for 48 hours (b) was performed. Green staining indicates the fluoresceinlabeled DNA in apoptotic cells and red signals indicates the propidium iodide cooperated non-apoptotic cells.

enhancement of TUNEL-positive cells was detected in ATRA-treated samples compared with control sample (Fig 2B). Previous studies have shown that expression of bcl-2 is frequently suppressed during apoptosis (Monczak et al., 1997), we therefore determined the expression of bcl-2 in response to ATRA-induced apoptosis by immunoblotting. As shown in Fig 3A, a decline in bcl-2 expression in ATRA-treated cells throughout the period of 24-72 hours 
A

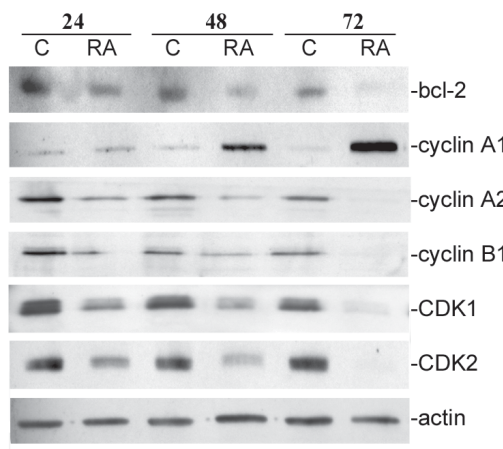

C

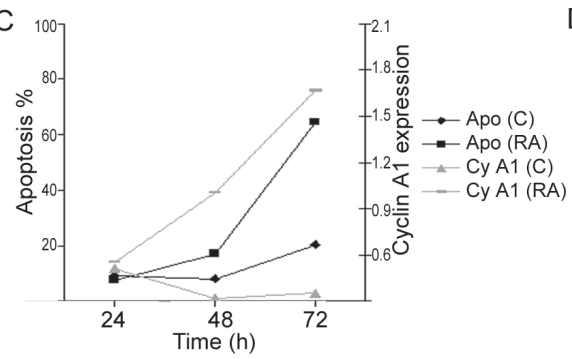

$\mathrm{E}$

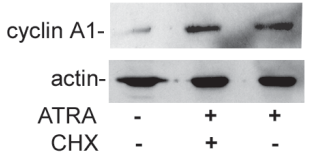

B Cyclin A1 expression in ATRA treated cells

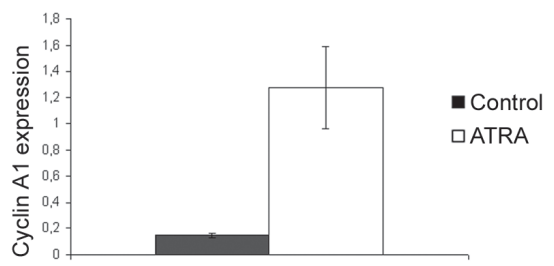

D Cyclin A1 mRNA in ATRA treated cells

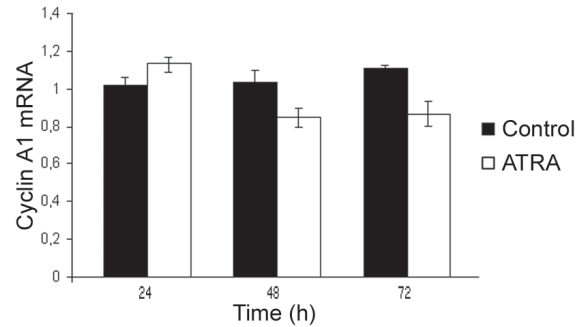

Figure 3. Expression of cell cycle regulatory proteins in response to ATRA-induced apoptosis. (A) Immunoblot analysis of cell cycle regulatory proteins in control and ATRA treated cells $(100 \mu \mathrm{M})$ for 24,48 and 72 hours. Control samples were indicated as C and ATRA-treated samples as RA. Cell lysates were prepared at the indicated times and subjected to $12 \%$ SDS-PAGE gel. The blots were probed with antibodies against bcl-2, cyclin A1, cyclin A2, cyclin B1, CDK1 and CDK2. Antibody to actin was used as a loading control. (B) Data presented are averages of three independent experiments to quantify the level of cyclin A1 after the treatment with ATRA for 72 hours. (C) Association between cyclin A1 expression and the rate of apoptosis. Darker lines represent proportion of apoptotic cells in controls (C) and ATRA-treated samples (RA). Lighter lines represent levels of cyclin A1 protein expression in controls (C) and ATRA-treated samples (RA). (D) Levels of cyclin A1 mRNA were determined by semi-quantitative RT-PCR. Data presented are averages of three independent experiments to quantify the mRNA levels. (E) Immunoblot analysis of control cells or cells treated with either ATRA alone or a combination of ATRA and CHX for 48 hours. 
was observed. Immunoblot analysis also revealed that the levels of cyclin A2, cyclin B1 and CDK1 and CDK2 began to decrease at 24 hours and were significantly reduced by 48 and 72 hours after exposure to ATRA (Fig 4). Conversely, the protein level of cyclin A1 increased upon induction of apoptosis in ATRA-treated cells (Fig $3 \mathrm{~A}$ and $3 \mathrm{~B}$ ). Similar results were obtained when using HL-60 and NB-4 cells using the same approaches (data not shown). The increased cyclin A1 expression throughout 24-72 hours of ATRA treatment correlated with a decrease in bcl-2 expression and increased rate of apoptosis (Fig 3C).

ATRA-induced apoptosis resulted in increased cyclin A1 protein levels but not $m R N A$ levels

We then investigated whether the increase in cyclin A1 expression in response to ATRA-induced apoptosis also occurred at mRNA level. Cyclin A1 mRNA expression was quantified in ATRA-treated and in control U-937 cells throughout the period of 24-72 hours. Semi-quantitative RT-PCR analysis from three independent experiments revealed that cyclin A1 mRNA levels remained virtually unchanged in ATRA-treated cells compared to control cells (Figure 3D). This was in striking contrast to what was observed for cyclin A1 protein expression and suggests that the increased cyclin A1 protein levels after ATRA treatment might be a result of post-transcriptional modifications.

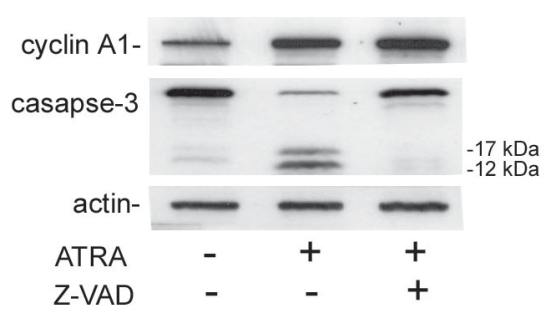

Figure 4. Expression of cyclin A1 in response to ATRA and caspase inhibitor treatment. Cells were treated with ATRA $(100 \mu \mathrm{M})$ alone or pre-treated with the caspase inhibitor Z-VAD-FMK. The protein expression of cyclin A1 and caspase-3 were analyzed using immunoblot. Antibody against actin was used as a loading control.

To further verify that the increased expression of cyclin A1 observed in ATRA-treated cells was indeed occurred at post-transcriptional level, we added cycloheximide (CHX), an agent that inhibit protein synthesis, in combination with ATRA to the cells. Immunoblot analysis revealed that there was a significant increase in cyclin A1 expression in ATRA-treated cells compared with that of control cells. Interestingly, the increase in cyclin A1 expression by ATRA remained unchanged in the presence of CHX. This further indicated that cyclin A1 expression might be regulated by post-transcriptional mechanisms in response to ATRAinduced apoptosis.

To investigate whether caspasemediated pathway is required for the increase in cyclin A1 protein expression, we added the non- 
reversible caspase inhibitor Z-VADFMK to the cells. The inhibitor completely blocked ATRA-induced activation of caspase-3. However, cyclin A1 expression was not suppressed by the addition of Z-VADFMK (Fig 4).

Induced overexpression of cyclin $A 1$ to $U$ 937 and T47D cells promoted apoptosis

Next, we asked whether cyclin A1 plays a role in the apoptotic process in U-937 cells. The effect of induced cyclin A1 expression on apoptosis was evaluated in U-937 cells transfected with cyclin A1-EGFP vector or EGFP control vector. Analysis from three independent transfections followed by Annexin- $\mathrm{V}$ staining of the cells revealed that there was a significant increase in the apoptotic fractions in cells transfected with cyclin A1-EGFP vector compared to that of cells transfected with EGFP control vector $(\mathrm{p}=0.022)$ (Fig 5A). TUNEL assay revealed that the rate of TUNELpositive cells in cyclin A1 transfected cells $(30 \%)$ was significantly higher compared with $10 \%$ in control EGFP vector transfected cells (Fig 5B). To ensure that the effect of cyclin A1 on apoptosis is specific, we transfected U937 cells with increased amount of vectors containing cyclin A1 cDNA and an increase in the fraction of apoptosis was observed (data not shown). There was an increase in cyclin A1 expression in cells transfected with cyclin A1-EGFP vector compared to the cells transfected with EGFP-control vector as measured by immunoblot analysis. It appeared that cyclin A1 protein level in cyclin A1 overexpressing cells was comparable to the endogenous level of cyclin A1 in cells treated with ATRA for 48 hours (Fig 5C). Although cells transfected with cyclin A1-EGFP vector do not produce fusion protein, those cells overexpressing cyclin A1 simultaneously express GFP protein. We monitored morphological changes of GFP positive cells undergoing apoptosis in cyclin A1-EGFP vector transfected cells and EGFP-control vector transfected cells using fluorescence microscopy. Control GFP expressing cells consist of viable cells (Fig 5D, panel a) and apoptotic cells which showed condensed, fragmented nuclei and reduction in volume, a typical characteristic of apoptotic cells (data not shown). In cyclin A1 expressing cells, cells with the characteristic of apoptosis were also detected (data not shown). To our surprise, we also observed cells with aberrant structures such as enlargement of cell volume and flattened morphology that were not present in control GFP expressing cells (Fig 5D, panel b) indicating that cyclin A1 induced apoptosis is also accompanied by aberrant enlargement of cells. 
A Apoptosis in cyclin A1 transfected U-937
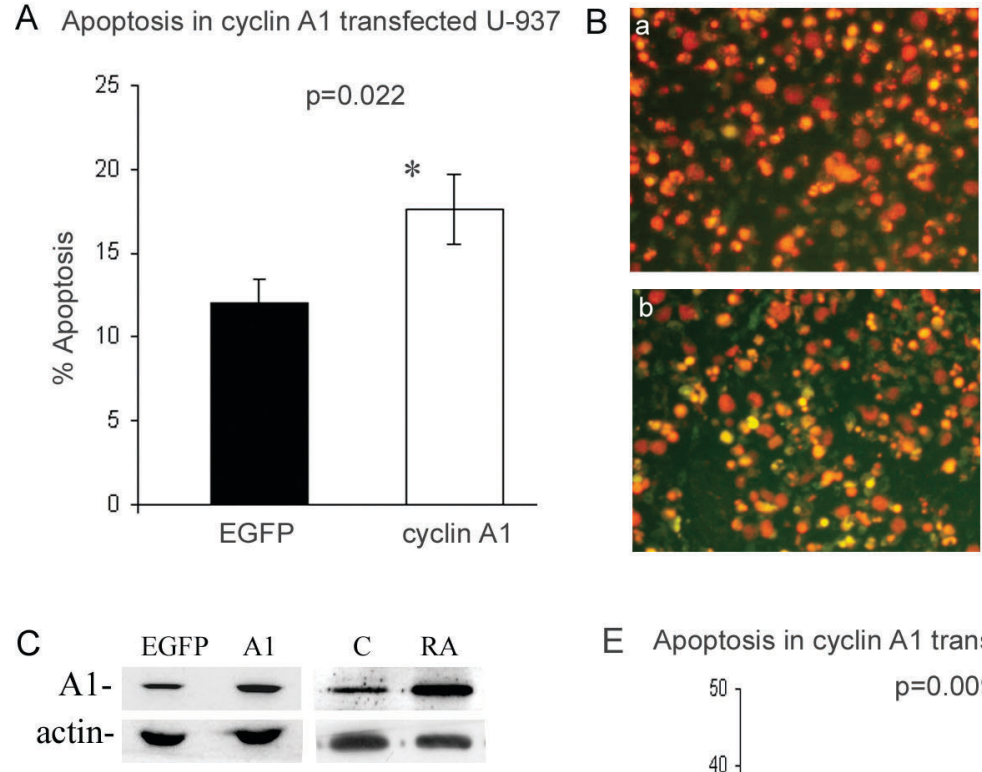

E Apoptosis in cyclin A1 transfected T47D

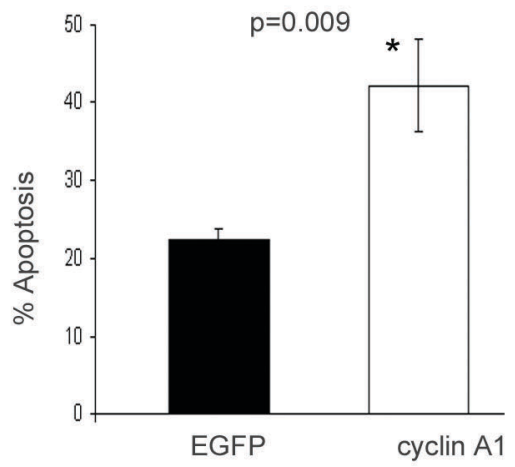

Figure 5. Effect of induced cyclin A1 overexpression on apoptosis in U-937 and T47D cells. (A) The proportion of apoptotic U-937 cells was measured with Annexin-V staining after transfection. The transfection efficiency was $54 \%$ for EGFP vector and $39 \%$ for cyclin A1-EGFP vector. Results are presented as mean rate of apoptosis \pm SD of three experiments performed in triplicates. (B) TUNEL assay of U-937 control (a) and cyclin A1 (b) transfected cells. (C) Immunoblot analysis of cyclin A1 protien levels in cyclin A1 overexpressing cells and cells treated with ATRA $(100 \mu \mathrm{M})$ for 48 hours. (D) Morphology of viable GFP positive U-937 cells in samples transfected with EGFPcontrol vector is shown in (a) and a cell with aberrant enlargement shown in (b) is from samples transfected with cyclin A1-EGFP vector. (E) The rate of apoptosis in T47D cells after transfection was analyzed using Annexin-V staining. The transfection efficiency was $73 \%$ for EGFP vector and $52 \%$ for cyclin A1-EGFP vector.

We further investigated whether the role of cyclin A1 in apoptosis was generalized to other cell line systems. Similar to what was observed in U-
937 cells, induction of overexpression of cyclin A1 in a breast cancer cell line, $\mathrm{T} 47 \mathrm{D}$ resulted in a significant increase in the rate of apoptosis as measured 
by Annexin-V staining followed by flow cytometry analysis (Fig 5E). Statistical analysis of the four different experiments revealed that T47D cells overexpressing cyclin A1 had significant higher portion of apoptotic population compared with EGFP vector expressing cells $(p=0.009)$. To further verify whether the ability of cyclin A1 to promote apoptosis is specific, we examined the effect of cyclin E on apoptosis. Similar to cyclin $\mathrm{A} 1$, cyclin $\mathrm{E}$ protein is also highly expressed in patients with AML (Iida et al., 1997; Radosevic et al., 2001). Under the same transfection conditions used for cyclin A1-EGFP vector, U-937 cells were transfected with cyclin E-EGFP vector and EGFP control vector. Cyclin E expression was induced into U-937 cells that lack endogenous cyclin E expression and the transfection efficiency was similar to what was achieved for the induction of cyclin A1 expression. In contrast to what was observed for cells overexpressing cyclin A1, there was no increased rate of apoptosis in cyclin E expressing cells as determined by the analysis from three independent experiments (data not shown). Further, no enhancement of apoptosis by cyclin $\mathrm{E}$ was detected at later time points of transfections (data not shown). This indicates that cyclin E was not able to initiate cell death.
Induced cyclin A1 expression enhance the apoptotic effect of ATRA

Since we have found that ATRAinduced apoptosis was coupled with increased expression of cyclin A1 protein and that cyclin A1 was able to promote apoptosis, we continued to investigate whether cyclin A1 was able to mediate ATRA-induced apoptosis in U-937 cells. GFP-control expressing cells and cyclin A1 expressing cells were treated with 50 $\mu \mathrm{M}$ ATRA for 48 hours and TUNEL

\section{A}

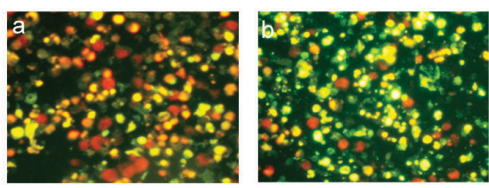

B

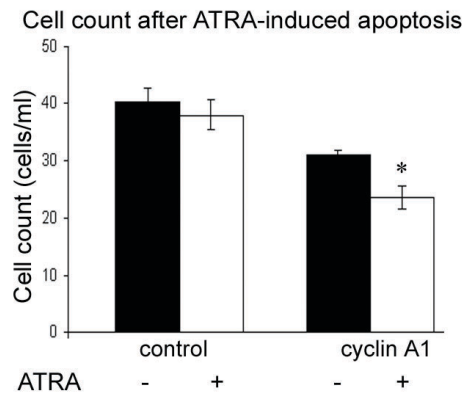

Figure 6. Effect of induced cyclin A1 overexpression on ATRA-induced apoptosis and cell growth inhibition on U-937 cells. (A) TUNEL staining of GFP control expressing cells treated with ATRA is shown in (a) and TUNEL staining of cyclin A1 expressing cells treated with ATRA is shown in (b). (B) The rate of cell number in GFP control expressing cells and in cyclin A1 expressing cells in the presence or absence of ATRA treatment. Results are presented as mean rate of cell count \pm SD of three experiments performed in triplicates. ${ }^{*} \mathrm{p}<0.05$. 
assay was performed to quantify the rate of apoptosis. ATRA caused much higher rate of apoptosis in cyclin A1 expressing cells than in GFP-control expressing cells (Fig 6A). Next we wanted to investigate whether cyclin A1 had ability to mediate ATRAinduced cell growth inhibition. Cell numbers were counted in cyclin A1 and GFP-control expressing cells treated with ATRA or treated with solvent. As shown in Figure 6B, cells overexpressing cyclin A1 is more sensitive to ATRA treatment, the numbers of cells overexpressing cyclin A1 in the presence of ATRA is significant decreased $(p=0.013)$ compared to control cells. This result suggests that cyclin A1 is able to enhance the effect of ATRA on the inhibition of cell growth.

\section{Discussion}

Previous studies have provided compelling evidence for a link between apoptosis and cell cycle proteins. For example, unscheduled activation of CDK molecules is associated with apoptosis induced by many chemotherapeutic drugs (Shimizu et al., 1995). It is also known that apoptosis-inducing agents inhibit the activities of CDKs, leading to eventual cell death (Dobashi et al., 2003). However, no studies have demonstrated a direct role of cell cycle proteins in contributing to apoptosis.
In the present study, we for the first time demonstrated a role for cyclin A1 in triggering apoptosis in both U-937 and T47D cells, whereas cyclin E, another important cell cycle regulator that also is involved in AML (Volm et al., 1997) did not initiate apoptosis. We found that cells overexpressing cyclin A1 and that underwent apoptosis showed typical apoptotic features including nuclear fragmentation, chromatin condensation and internal chromosomal DNA cleavage as detected by TUNEL assay. Further, cyclin A1 promoted apoptosis in U937 cells that do not express functional p53 (Rizzo et al., 1998), suggesting that cyclin A1 function in this context does not seem to relate to p53-mediated pathways. We have shown that a high cellular concentration of cyclin A1 induces apoptosis. The expression level of cyclin A1 protein in cyclin A1 overexpressing cells was comparable to its endogenous level in cells treated with ATRA. However, the ability of cyclin A1 to induce apoptosis is less pronounced when compared to the apoptosis inducing capability of the pro-apoptotic factors PML and BAX that has been shown in previously studies (Xiang et al., 1996; Quignon et al., 1998). Moreover, given that cyclin A1 has the ability to enhance ATRAinduced apoptosis, we propose that the potential role of cyclin A1 in apoptosis would be to influence one of the apoptosis-inducing pathways. We have recently demonstrated that 
cyclin A1 interacts with RAR $\alpha$ following ATRA treatment of leukemic cells (Ekberg et al., 2004). It would be interesting to investigate whether the apoptosis promoting activity of cyclin A1 on ATRA-induced apoptosis is due to its interaction with a major ATRA receptor RAR $\alpha$. Taken together, the expression pattern of cyclin A1 is linked to its function in mediating ATRA-induced cell cycle arrest and apoptosis.

ATRA is widely used as an anti-tumor agent, particularly for targeting leukemic cells (He et al., 1999). Although it is known that ATRA induces terminal differentiation and apoptosis, the precise mechanisms underlying ATRA induced apoptosis are poorly understood. Our result indicated that sustained treatment with ATRA appeared to be necessary for the induction of apoptosis. Further, ATRA-induced apoptosis is accompanied by activation of casepase- 3 and down-regulation of bcl-2 at the time of induction of apoptosis. This suggests that the apoptotic pathways involving caspase-3 and bcl-2 might be associated with ATRA-induced apoptosis.

Studying the cell cycle regulatory proteins involved in ATRA-induced apoptosis of hematopoietic cells is important for understanding the molecular events underlying the action of ATRA. Our present study has demonstrated that changes of the expression of cyclin A1 and the other cell cycle regulatory proteins including cyclin A2, cyclin B1, CDK1 and CDK2 is a result of apoptosis induced by ATRA. Down regulation of myc has been suggested to be an early and important event in ATRA induced cell cycle arrest, leading to a downregulation of cyclin $\mathrm{E}$ and eventually cyclin A2 and cyclin B expression (Dimberg et al., 2002). Further, upregulation of p21 and p27 induced by ATRA may also contribute to a declined expression of cyclins and decreased activity of CDKs (Dimberg et al., 2002). It has also been shown that expression of several cell cycle regulators such as p21, p27, CDK1 and $\mathrm{pRB}$ is regulated by caspases during apoptosis (Vermeulen et al., 2003). Thus, ATRA may actively halt cell cycle progression leading to the declined expression of cyclins and CDKs during the process of apoptosis.

One of our striking observations is that the level of cyclin A1 protein expression was significantly increased in cells undergoing apoptosis induced by ATRA, which was in contrast to that of the other cell cycle regulatory proteins analyzed. This suggests that an increase in cyclin A1 expression in response to ATRA is unique. Expression of cyclin A1 is restricted to 
a few normal human tissues including testis, bone marrow and brain. In normal bone marrow, cyclin A1 expression is restricted to hematopoietic progenitor cells (Yang et al., 1999). Further, abnormal expression of cyclin A1 initiated acute myeloid leukemia in transgenic mice (Liao et al., 2001). These correlated studies indicate that cyclin A1 may have function in differentiation and apoptosis in hematopoietic cells. In response to ATRA-treatment, cyclin A1 appeared to have similar expression patterns to that of several pre-apoptotic factors, such as PML and Bim. Both PML and Bim have been found to have an increased expression in response to cell death induced by different apoptosis-inducing reagents such as FAS and TNF $\alpha$ (Bernardi \& Pandolfi, 2003; Jin et al., 2001; Wang et al., 1998; Zhang \& Insel, 2004). In the present study, the global inhibition of caspase activity by ZVAD in order to prevent ATRA-mediated apoptosis did not suppress cyclin A1 expression. This suggests that increased cyclin A1 expression was not a secondary effect of ATRA-induced apoptosis. In addition, we demonstrated that cyclin A1 protein expression but not mRNA expression increased in response to ATRA-induced apoptosis. Further, the increased cyclin A1 protein expression induced by ATRA treatment was not affected when protein synthesis was blocked by CHX. These results suggest that the concomitant increase in cyclin
A1 protein expression in response to ATRA at dose- and time-dependent fashion is not a result of overexpression of cyclin A1 mRNA, but may be due to protein stabilization or modification by posttranscriptional mechanisms. Posttranscriptional regulation via the proteasome pathway has previously been reported to be an important mechanism for the regulation of cyclin D1 in cells arrested at G1 phase and in apoptotic cells induced by TNF $\alpha(\mathrm{Hu}$ et al., 2002). Our finding may be analogous to the regulation of cyclin $\mathrm{D} 1$ in response to TNF $\alpha$ treatment.

Elevated levels of cyclin A1 have been observed in AML patients and several leukemic cell lines (Yang et al., 1999). Further, cyclin A1 is one of the causative factors for transformation as shown in transgenic mice model (Liao et al., 2001). Our present study showing the ability of cyclin A1 to induce apoptosis seems to be in conflict with its role in proliferation. However, the discrepancies between the previous studies and our present study could reflect that cyclin A1 might function distinctively under different conditions. Our recent studies have demonstrated that the alteration of subcellular localization of cyclin A1 correlated with the leukemic phenotype and further, with the absence of complexes between cyclin $\mathrm{A} 1 / \mathrm{CDK} 1$, and these results provided 
evidence suggesting that in addition to the elevated levels of cyclin A1, its abnormal cytoplasmic localization might be involved in the transformation process (Ekberg et al., 2004). Our finding may be analogous to underlying mechanisms of CDK inhibitors in apoptosis and proliferation. For example, p21 and p27 can either induce apoptosis or protect cells from apoptosis, depending on cellular context (Blagosklonny, 2002; El-Deiry, 2001; Eymin et al., 1999; Liang et al., 2002; Viglietto et al., 2002; Zhou et al., 2001). Ours and others studies suggest a new mechanism of cyclin- or CDK inhibitor-related cellular transformation and apoptosis.

In conclusion, our results indicate that cyclin A1 may have a role in apoptosis and specifically in ATRA induced apoptosis. Investigation of the cellular response that confers specificity of ATRA treatment will increase the understanding of the mechanisms underlying leukemogenesis.

\section{Acknowledgements}

We thank Elise Nilsson for technical assistant, Dr. Debra Wolgemuth (Department of Genetics and Development, Columbia University, New York) and Dr. Sven Påhlman (Department of Laboratory Medicine, Division of Molecular Medicine,
Malmö) for helpful discussions. This work was supported by the Swedish National Research Council, the Swedish Cancer Society, the Swedish Children Cancer Foundation, MAS Cancer Fondation, Kunlig Fysiografisk Sällskapet in Lund, Crafoordska stiftelsen, and MAS Fondation to JPL, the Government Public Health Grant (ALF) and Swegene/WCN to GL.

\section{References}

Bernardi, R., \& Pandolfi, P. P. (2003). Role of PML and the PML-nuclear body in the control of programmed cell death. Oncogene, 22, 9048-9057.

Blagosklonny, M. V. (2002). Are p27 and p21 cytoplasmic oncoproteins? Cell Cycle, 1, 391-393.

Cardoso, M. C., Leonhardt, H., \& Nadal-Ginard, B. (1993). Reversal of terminal differentiation and control of DNA replication: cyclin A and Cdk2 specifically localize at subnuclear sites of DNA replication. Cell, 74, 979-992.

Dao, M., \& Nolta, J. (1999). Molecular control of cell cycle progression in primary human hematopoietic stem cells: methods to increase levels of retroviral-mediated transduction. Leukemia, 13, 1473-1480.

Dimberg, A., Bahram, F., Karlberg, I., Larsson, L. G., Nilsson, K., \& Oberg, F. (2002). Retinoic acid-induced cell cycle arrest of human myeloid cell lines is associated with sequential 
down-regulation of c-Myc and cyclin

E and posttranscriptional up-

regulation of p27(Kip1). Blood, 99, 2199-2206.

Dobashi, Y., Takehana, T., \& Ooi, A. (2003). Perspectives on cancer therapy: cell cycle blockers and perturbators. Curr Med Chem, 10, 2549-2558.

Ekberg, J., Landberg, G., Holm, C., Richter, J., Wolgemuth, D. J., \& Persson, J. L. (2004). Regulation of the cyclin A1 protein is associated with its differential subcellular localization in hematopoietic and leukemic cells. Oncogene, 23, 90829089.

El-Deiry, W. S. (2001). Akt takes centre stage in cell-cycle deregulation. Nat Cell Biol, 3, E71-73.

Eymin, B., Haugg, M., Droin, N., Sordet, O., Dimanche-Boitrel, M. T., \& Solary, E. (1999). p27Kip1 induces drug resistance by preventing apoptosis upstream of cytochrome c release and procaspase- 3 activation in leukemic cells. Oncogene, 18, 1411-1418.

Fujimura, S., Suzumiya, J., Yamada, Y., Kuroki, M., \& Ono, J. (2003). Downregulation of Bcl-xL and activation of caspases during retinoic acid-induced apoptosis in an adult T-cell leukemia cell line. Hematol J, 4, 328-335.

Furukawa, Y. (1998). Cell cycle regulation of hematopoietic stem cells. Hum Cell, 11, 81-92.
He, L. Z., Merghoub, T., \& Pandolfi, P. P. (1999). In vivo analysis of the molecular pathogenesis of acute promyelocytic leukemia in the mouse and its therapeutic implications. Oncogene, 18, 5278-5292.

$\mathrm{Hu}, \mathrm{X} .$, Bryington, M., Fisher, A. B., Liang, X., Zhang, X., Cui, D., Datta, I., \& Zuckerman, K. S. (2002). Ubiquitin/proteasome-dependent degradation of D-type cyclins is linked to tumor necrosis factorinduced cell cycle arrest. J Biol Chem, 277, 16528-16537.

Iida, H., Towatari, M., Tanimoto, M., Morishita, Y., Kodera, Y., \& Saito, H. (1997). Overexpression of cyclin E in acute myelogenous leukemia. Blood, 90, 3707-3713.

Jin, M. L., Zhang, P., Ding, M. X., Yun, J. P., Chen, P. F., Chen, Y. H., \& Chew, Y. Q. (2001). Altered expression of nuclear matrix proteins in etoposide induced apoptosis in HL-60 cells. Cell Res, 11, 125-134.

Jin, Y. H., Yim, H., Park, J. H., \& Lee, S. K. (2003). Cdk2 activity is associated with depolarization of mitochondrial membrane potential during apoptosis. Biochem Biophys Res Commun, 305, 974-980.

Liang, J., Zubovitz, J., Petrocelli, T., Kotchetkov, R., Connor, M. K., Han, K., Lee, J. H., Ciarallo, S., Catzavelos, C., Beniston, R., Franssen, E., \& Slingerland, J. M. (2002). PKB/Akt phosphorylates p27, impairs nuclear import of p27 and 
opposes p27-mediated G1 arrest.

Nat Med, 8, 1153-1160.

Liao, C., Li, S. Q., Wang, X., Muhlrad, S., Bjartell, A., \& Wolgemuth, D. J. (2004). Elevated levels and distinct patterns of expression of A-type cyclins and their associated cyclindependent kinases in male germ cell tumors. Int J Cancer, 108, 654-664.

Liao, C., Wang, X. Y., Wei, H. Q., Li, S. Q., Merghoub, T., Pandolfi, P. P., \& Wolgemuth, D. J. (2001). Altered myelopoiesis and the development of acute myeloid leukemia in transgenic mice overexpressing cyclin A1. Proc Natl Acad Sci U S A, 98, 6853-6858.

Liu, D., Liao, C., \& Wolgemuth, D. J. (2000). A role for cyclin A1 in the activation of MPF and G2-M transition during meiosis of male germ cells in mice. Dev Biol, 224, 388400.

Liu, D., Matzuk, M. M., Sung, W. K., Guo, Q., Wang, P., \& Wolgemuth, D. J. (1998). Cyclin A1 is required for meiosis in the male mouse. Nat Genet, 20, 377-380.

Loden, M., Stighall, M., Nielsen, N. H., Roos, G., Emdin, S. O., Ostlund, H., \& Landberg, G. (2002). The cyclin D1 high and cyclin E high subgroups of breast cancer: separate pathways in tumorogenesis based on pattern of genetic aberrations and inactivation of the pRb node. Oncogene, 21, 46804690.

Mehta, K., McQueen, T., Neamati, N., Collins, S., \& Andreeff, M. (1996).
Activation of retinoid receptors RAR alpha and RXR alpha induces differentiation and apoptosis, respectively, in HL-60 cells. Cell Growth Differ, 7, 179-186.

Monczak, Y., Trudel, M., Lamph, W. W., \& Miller, W. H., Jr. (1997). Induction of apoptosis without differentiation by retinoic acid in PLB-985 cells requires the activation of both RAR and RXR. Blood, 90, 3345-3355.

Ongkeko, W., Ferguson, D. J., Harris, A. L., \& Norbury, C. (1995). Inactivation of Cdc2 increases the level of apoptosis induced by DNA damage. J Cell Sci, 108 (Pt 8), 2897-2904.

Passegue, E., Jamieson, C. H., Ailles, L. E., \& Weissman, I. L. (2003). Normal and leukemic hematopoiesis: are leukemias a stem cell disorder or a reacquisition of stem cell characteristics? Proc Natl Acad Sci U $S$ A, 100 Suppl 1, 11842-11849.

Puccetti, E., \& Ruthardt, M. (2004). Acute promyelocytic leukemia: PML/RARalpha and the leukemic stem cell. Leukemia, 18, 1169-1175.

Quignon, F., De Bels, F., Koken, M., Feunteun, J., Ameisen, J. C., \& de The, H. (1998). PML induces a novel caspase-independent death process. Nat Genet, 20, 259-265.

Radosevic, N., Delmer, A., Tang, R., Marie, J. P., \& AjchenbaumCymbalista, F. (2001). Cell cycle regulatory protein expression in fresh acute myeloid leukemia cells and after drug exposure. Leukemia, 15, 559-566. 
Rizzo, M. G., Zepparoni, A.,

Cristofanelli, B., Scardigli, R.,

Crescenzi, M., Blandino, G.,

Giuliacci, S., Ferrari, S., Soddu, S., \&

Sacchi, A. (1998). Wt-p53 action in

human leukaemia cell lines

corresponding to different stages of differentiation. Br J Cancer, 77, 14291438.

Roberts, J. M. (1999). Evolving ideas about cyclins. Cell, 98, 129-132.

Shimizu, T., O'Connor, P. M., Kohn, K.

W., \& Pommier, Y. (1995).

Unscheduled activation of cyclin B1/

Cdc2 kinase in human

promyelocytic leukemia cell line

HL60 cells undergoing apoptosis

induced by DNA damage. Cancer Res, 55, 228-231.

Tewari, M., Quan, L. T., O'Rourke, K.,

Desnoyers, S., Zeng, Z., Beidler, D.

R., Poirier, G. G., Salvesen, G. S., \&

Dixit, V. M. (1995). Yama/CPP32 beta, a mammalian homolog of CED-3, is

a CrmA-inhibitable protease that

cleaves the death substrate

poly(ADP-ribose) polymerase. Cell, 81, 801-809.

Wang, Z. G., Ruggero, D., Ronchetti, S., Zhong, S., Gaboli, M., Rivi, R., \& Pandolfi, P. P. (1998). PML is essential for multiple apoptotic pathways. Nat Genet, 20, 266-272.

Vermeulen, K., Berneman, Z. N., \& Van Bockstaele, D. R. (2003). Cell cycle and apoptosis. Cell Prolif, 36, 165-175.

Viglietto, G., Motti, M. L., Bruni, P., Melillo, R. M., D'Alessio, A., Califano, D., Vinci, F., Chiappetta,
G., Tsichlis, P., Bellacosa, A., Fusco,

A., \& Santoro, M. (2002).

Cytoplasmic relocalization and inhibition of the cyclin-dependent kinase inhibitor p27(Kip1) by PKB/ Akt-mediated phosphorylation in breast cancer. Nat Med, 8, 1136-1144. Volm, M., Koomagi, R., Stammler, G., Rittgen, W., Zintl, F., \& Sauerbrey, A. (1997). Prognostic implications of cyclins (D1, E, A), cyclin-dependent kinases (CDK2, CDK4) and tumorsuppressor genes (pRB, p16INK4A) in childhood acute lymphoblastic leukemia. Int J Cancer, 74, 508-512.

Xiang, J., Chao, D. T., \& Korsmeyer, S. J. (1996). BAX-induced cell death may not require interleukin 1 beta-converting enzyme-like proteases. Proc Natl Acad Sci U S A, 93, 14559-14563.

Yang, R., Nakamaki, T., Lubbert, M., Said, J., Sakashita, A.,

Freyaldenhoven, B. S., Spira, S., Huynh, V., Muller, C., \& Koeffler, H. P. (1999). Cyclin A1 expression in leukemia and normal hematopoietic cells. Blood, 93, 2067-2074.

Zhang, L., \& Insel, P. A. (2004). The proapoptotic protein Bim is a convergence point for $\mathrm{cAMP} /$ protein kinase A- and glucocorticoidpromoted apoptosis of lymphoid cells. J Biol Chem, 279, 20858-20865.

Zhou, B. P., Liao, Y., Xia, W., Spohn, B., Lee, M. H., \& Hung, M. C. (2001). Cytoplasmic localization of p21Cip1/ WAF1 by Akt-induced phosphorylation in HER-2/neuoverexpressing cells. Nat Cell Biol, 3, 245-252. 